\title{
Perfil dos pacientes atendidos no Centro de Traumatismo em Odontologia frente às fraturas coronárias e suas sequelas
}

\author{
Profile of patients taken in the Center of Dental Traumatism \\ in relation to coronary fractures and their sequels \\ Perfil de los pacientes atendidos en el Centro de Traumatismo Odontológico en \\ relación a las fracturas coronarias y sus secuelas \\ Fernanda Chiguti YAMASHITA ${ }^{1}$ \\ Isabela Chiguti YAMASHITA ${ }^{2}$ \\ Nair Narumi Orita PAVAN \\ Marcos Sérgio ENDO ${ }^{3}$
}

${ }^{1}$ Doutoranda em Odontologia Integrada - Programa de Pós-Graduação em Odontologia Integrada, Departamento de Odontologia (DOD), Centro de Ciências da Saúde (CCS), Universidade Estadual de Maringá (UEM), 87080-000, Maringá - PR, Brasil

${ }^{2}$ Mestre em Clínica Odontológica - Programa de Pós-Graduação em Clínica Odontológica, Centro de Ciências da Saúde Universidade Federal do Espírito Santo (UFES), 29043-900, Vitória - ES, Brasil

${ }^{3}$ Professor Adjunto em Endodontia - Departamento de Odontologia (DOD), Centro de Ciências da Saúde (CCS), Universidade Estadual de Maringá (UEM), 87080-000, Maringá - PR, Brasil

\begin{abstract}
Resumo
O traumatismo dentário é um evento bastante comum, representando uma situação de urgência que compreende questões físicas e psicológicas. $\mathrm{O}$ objetivo deste trabalho foi determinar a prevalência das fraturas coronárias em dentes permanentes e também uma possível complicação, como a necrose pulpar, decorrente do traumatismo dentário. Este estudo transversal e retrospectivo foi realizado por meio da avaliação dos prontuários e exames radiográficos de um centro de referência em traumatismos dentários na cidade de Maringá-PR, entre os períodos de janeiro de 2008 a dezembro de 2014. Os dados demográficos de interesse foram: sexo, idade do paciente no momento da injúria traumática, dente acometido, causa, tipo da injúria traumática, estágio de desenvolvimento radicular e presença de necrose pulpar. $\mathrm{O}$ sexo masculino (68\%) foi mais acometido que o sexo feminino (32\%), sendo que as injúrias ocorreram mais frequentemente nos pacientes com idade entre 6-11 anos (60\%). O incisivo central superior foi o dente mais afetado $(74 \%)$ e a injúria mais frequente foi fratura de esmalte e dentina sem exposição pulpar (70\%). A necrose pulpar foi observada em $14 \%$ das fraturas coronárias. O teste exato de Fisher não demonstrou uma associação entre a necrose pulpar e as fraturas coronárias $(\mathrm{p}=0,1153)$ e entre a necrose pulpar e o estágio de desenvolvimento radicular $(\mathrm{p}=0,191)$. Conclui-se que a prevalência de fraturas coronárias foi maior em crianças e adolescentes. Uma frequência pequena de necrose pulpar foi observada e não se observou uma associação com as fraturas coronárias e o estágio de desenvolvimento radicular.

Descritores: Traumatismos Dentários; Prevalência; Necrose da Polpa Dentária.
\end{abstract}

\begin{abstract}
Dental trauma is a common event, representing an urgency situation that includes physical and psychological issues. The aim of this study was to determine the prevalence of crown fractures in permanent teeth and also a possible complication, such as pulp necrosis, due to dental trauma. This cross-sectional and retrospective study was performed through the evaluation of medical records and radiographs of a reference centre in dental trauma in the city of Maringá, between January 2008 and December 2014. The demographic interest data were: sex, age of the patient at the moment of traumatic injury, tooth affected, cause, type of traumatic injury, stage of root development and presence of pulp necrosis. The male gender (68\%) was more affected than the female gender $(32 \%)$, and injuries occurred more frequently in patients aged 6-11 years $(60 \%)$. The upper central incisor was the most affected tooth (74\%) and the most frequent injury was enamel and dentin fracture without pulp exposure (70\%). Pulp necrosis was observed in $14 \%$ of the crown fractures. Fisher's exact test did not demonstrate an association between pulpal necrosis and crown fractures $(p=0.1353)$ and between pulpal necrosis and root development stage $(p=0.191)$. These findings suggest that the prevalence of crown fractures was higher in children and adolescents. A small frequency of pulp necrosis was observed and there was no association with crown fractures and the stage of root development.
\end{abstract} Descriptors: Tooth Injuries; Prevalence; Dental Pulp Necrosis

\section{Resumen}

El traumatismo dentario es un evento bastante común, representando una situación de urgencia involucrando aspectos físicos y psicológicos. El objetivo de este estudio fue determinar la prevalencia de fracturas coronarias en dientes permanentes y también una posible complicación, como la necrosis pulpar, debido al traumatismo dentario. Este estudio retrospectivo, transversal fue realizado por medio de la evaluación de fichas clínicas y exámenes radiográficos de un centro de referencia en traumatismos dentarios en la ciudad de Maringá-PR, desde enero del 2008 a diciembre del 2014. Los datos demográficos de interés fueron: sexo, edad del paciente en el momento del traumatismo dentario, diente afectado, causa, tipo de lesión traumática, etapa de desarrollo radicular y presencia de necrosis pulpar. El sexo masculino (68\%) fue más afectado que el sexo femenino (32\%), y las lesiones traumáticas se presentaron con más frecuencia en pacientes de 6-11 años de edad (60\%). El incisivo central superior fue el diente más afectado (74\%) y la lesión traumática más frecuente fue fractura de esmalte y dentina sin exposición pulpar (70\%). La necrosis pulpar fue observada en 14\% de las fracturas coronarias. La prueba exacta de Fisher no mostró una asociación entre la necrosis pulpar y las fracturas coronarias $(\mathrm{p}=0,1153)$ y entre la necrosis pulpar y la etapa de desarrollo radicular $(\mathrm{p}=0,191)$. Estos resultados sugieren que la prevalencia de fracturas coronarias fue mayor en niños y adolescentes. Una baja frecuencia de necrosis pulpar fue observada, no observando una asociación con las fracturas coronarias y la etapa de desarrollo radicular.

Descriptores: Traumatismos de los Dientes; Prevalencia; Necrosis de la Pulpa Dental

\section{INTRODUÇÃO}

O traumatismo alvéolo dentário é considerado uma injúria decorrente do impacto nos dentes, exigindo intervenção imediata devido à sua natureza urgencial, uma vez que sua ocorrência influencia na função e estética do indivíduo ${ }^{1}$.
A prevalência do traumatismo dentário em crianças e adolescentes menores de 18 anos de idade é de $17,5 \%$, com variações entre as diferentes regiões geográficas ${ }^{2}$ sendo o sexo masculino mais acometido que o feminino ${ }^{3,4}$. As injúrias traumáticas em geral afetam os dentes permanentes 5 
e são decorrentes de acidentes de bicicletas, quedas ou práticas esportivas ${ }^{6}$

As fraturas de esmalte e as fraturas de esmalte e dentina também são denominadas de fraturas coronárias não complicadas. As fraturas de esmalte são injúrias confinadas somente no esmalte dentário e apresentam um bom prognóstico pulpar, isso quando não são associadas com luxações ${ }^{7}$. Já nas injúrias em que a dentina está exposta, uma variedade de bactérias e toxinas podem se difundir através dos túbulos dentinários até a polpa dentária, e assim podem provocar alterações inflamatórias no complexo dentinapolpa ${ }^{8}$.

Fraturas coronárias complicadas envolvem fraturas no esmalte, dentina e polpa, em que a exposição da polpa fornece uma via para entrada de bactérias. Logo após o traumatismo, a polpa exposta é coberta por uma camada de fibrina e uma zona de inflamação aguda pode ser observada imediatamente subjacente ao local da exposição9. Nestes casos, para avaliação das alterações pulpares, um tempo mínimo de 3 meses de acompanhamento pós-operatório deve ser considerado ${ }^{10}$.

A necrose pulpar é considerada a complicação mais comum pós-trauma ${ }^{11}$. A mesma pode ocorrer devido a um rompimento ou lesão do feixe vascular e nervoso no forame apical, também denominada de necrose asséptica, ou devido a invasão bacteriana relacionada a necrose por liquefação ${ }^{12}$ Tem sido demonstrado que o desenvolvimento de necrose pulpar pode ser dependente do tipo da injúria e do estágio de desenvolvimento da raiz no momento do trauma ${ }^{13,14}$. Quando o exame é realizado poucos dias após o traumatismo dentário, o teste de sensibilidade pulpar poderá resultar em falso negativo. Esta perda temporária de resposta é causada por uma lesão, inflamação, pressão ou tensão das fibras nervosas apicais e pode-se levar 8 semanas, ou mais tempo, para se obter uma resposta normal da polpa dentária ${ }^{15}$. Dessa maneira, ao fazer o diagnóstico, o cirurgião-dentista deve estar ciente do processo da dinâmica que envolve a polpa.

O objetivo deste trabalho foi determinar a prevalência das fraturas coronárias em dentes permanentes e também uma possível complicação, como a necrose pulpar, decorrente do traumatismo dentário.

\section{MATERIAL E MÉTODO}

Esta pesquisa foi aprovada pelo Comitê de Ética em Pesquisa (CEP) da Universidade Estadual de Maringá (CAAE n. 53207716.5.0000.0104).

Este estudo transversal e retrospectivo foi realizado por meio da avaliação dos prontuários e exames radiográficos de um centro de referência em traumatismos dentários na cidade de Maringá-PR, o projeto de Extensão Centro Especializado Maringaense de Traumatismo em Odontologia (CEMTrau/Odonto), na Universidade Estadual de Maringá (UEM) entre os períodos de janeiro de 2008 a dezembro de 2014. Os dados demográficos de interesse foram: sexo, idade do paciente no momento da injúria traumática, dente acometido, causa e tipo da injúria traumática. Informações referentes ao estágio de desenvolvimento radicular e presença de necrose pulpar também foram coletados. As informações contidas nos prontuários juntamente com as radiografias periapicais foram analisadas por um avaliador, com auxílio de uma lupa e um negatoscópio.

Traumatismos aos tecidos periodontais e ao osso alveolar, fraturas radiculares e corono-radiculares, dentes que já haviam sido submetidos ao tratamento endodôntico, prontuários com ausência de radiografias e sem informações a respeito do trauma foram excluídos da pesquisa.

O diagnóstico da necrose pulpar foi baseado nos seguintes critérios clínicos e radiográficos: ausência de sensibilidade pulpar, alteração de cor da coroa dentária, radioluscência apical, presença de fístula ou ausência de dor no teste de cavidade ${ }^{16,17}$.

Os dados obtidos foram digitalizados em uma planilha do programa Microsoft Excel 2013 e analisados estatisticamente com auxílio do Software $R$ versão 3.1. Foi realizada estatística descritiva dos dados. O teste exato de Fisher foi utilizado para avaliar a possível associação da necrose pulpar com as fraturas coronárias e o estágio de desenvolvimento radicular $(\mathrm{p}<0,05)$.

\section{RESULTADOS}

A população de traumatismos com danos dentários foi composta de 99 pacientes, em que foram examinados 146 dentes. A prevalência de traumatismo dentário no sexo masculino foi de $68 \%$ e no sexo feminino foi de $32 \%$. A idade dos pacientes variou de 6-56 anos, sendo que as injúrias ocorreram mais frequentemente nos pacientes com idade entre 6-11 anos (60\%).

A injúria mais frequente foi a fratura de esmalte e dentina, com 102 observações (70\%). Fratura de esmalte e dentina com exposição pulpar $(23 \%)$ e fratura de esmalte (11\%) foram menos observadas. $\mathrm{O}$ incisivo central superior foi o dente mais afetado (74\%), seguido do incisivo lateral superior (14\%) e incisivos centrais e laterais inferiores $(5 \%)$. A causa mais comumente reportada destes traumatismos foi a queda, que contribuíram para $36 \%$ dos casos, seguido por $21 \%$ de acidentes por bicicleta. Acidente motociclístico, colisão, esporte contribuíram com $8 \%$ dos casos. Acidente automobilístico, agressão física, atropelamento e piscina contribuíram cada uma com menos ou igualmente a $5 \%$. Ainda, $3 \%$ dos prontuários não reportavam a causa da injúria dentária.

Entre as possíveis sequelas, a necrose pulpar foi observada somente em 20 dentes (14\%) (Tabela 1). Sendo que 17 apresentaram ápice fechado e 3 apresentaram ápice aberto no momento do trauma. Não foi observada uma diferença estatisticamente significante entre as diferentes fraturas coronárias e a necrose pulpar $(\mathrm{p}=0,1153)$ e também entre o estágio de desenvolvimento radicular e a necrose pulpar $(\mathrm{p}=0,191)$.

Tabela1. Número de dentes que apresentaram necrose pulpar em relação ao tipo de injúria

\begin{tabular}{|c|c|}
\hline Injúria & Necrose pulpar \\
\hline Fratura de esmalte & 0 \\
\hline Fratura de esmalte e dentina & 12 \\
\hline $\begin{array}{l}\text { Fratura de esmalte e dentina } \\
\text { com exposição pulpar }\end{array}$ & 8 \\
\hline
\end{tabular}

\section{DISCUSSÃO}

As informações referentes à epidemiologia estão de acordo com dados da literatura científica. $\mathrm{O}$ resultado deste estudo confirmou que ser do sexo masculino é um fator predisponente relacionado a ocorrência de injúrias dentárias ${ }^{1}$. O dente mais vulnerável foi o incisivo central superior e a fratura envolvendo o esmalte e a dentina foi a injúria que apresentou maior frequência corroborando com estudos anteriores ${ }^{18,19,20}$. 
Normalmente a necrose pulpar nas fraturas coronárias não complicadas é rara. No presente estudo, a necrose pulpar nas fraturas de esmalte não foi observada e nas fraturas de esmalte e dentina a prevalência foi de $12 \%$, o que demonstra consistência com estudos anteriores, que encontraram uma prevalência de 0-3,5\% em fraturas de esmalte e 4 a $22 \%$ nas fraturas envolvendo a dentina ${ }^{21,22,23}$. Ainda, Wang et al. ${ }^{23}$ (2014) reportaram o aumento de até $40 \%$ de necrose pulpar quando não há proteção da dentina exposta, uma vez que a proteção auxilia no processo de cicatrização pulpar repelindo fatores químico-físicos que podem induzir a necrose da polpa.

O prognóstico da sobrevivência pulpar nas fraturas coronárias complicadas varia de $63 \%$ a $98 \%$ e parece ser favorável quando se utiliza terapias pulpares conservadoras. A necrose pulpar nas fraturas com exposição pulpar foi observada em $24 \%$ dos dentes que sofreram trauma, concordando com os trabalhos de Hecova et al. ${ }^{22}$ (2010). Os mesmos autores demonstraram que o método de tratamento influencia no aparecimento da necrose pulpar, onde o capeamento pulpar resultou em uma alta prevalência de necrose pulpar $(45,5 \%)$ quando comparado com a pulpotomia parcial $(13,6 \%)$.

O estágio de desenvolvimento radicular é um dos fatores que mais influenciam no aparecimento ou não de sequelas. Dentes com ápice aberto apresentam um prognóstico melhor quando comparados com dentes com ápice fechado ${ }^{24}$. Essa associação pode ser explicada através do diâmetro do forame apical no momento da injúria. Isto é, com um aumento do diâmetro do forame apical, a frequência de necrose pulpar diminuiu ${ }^{17}$. O presente estudo não demonstrou uma associação entre a necrose pulpar e o estágio de desenvolvimento radicular $(p=0,191)$, entretanto dentes imaturos apresentaram uma frequência menor de necrose pulpar. Fatores como ápice aberto e um suprimento neurovascular apical maior de dentes permanentes jovens podem acabar ajudando a prevenir uma invasão bacteriana ${ }^{25}$.

\section{CONCLUSÃO}

As fraturas coronárias ocorreram com maior frequência no sexo masculino, sendo que a injúria mais comumente encontrada foi a fratura de esmalte e dentina. Uma baixa frequência de necrose foi observada, e não foi demonstrada associação entre esta complicação e as fraturas coronárias. Também não se observou associação entre a necrose pulpar e o estágio de desenvolvimento radicular.

Campanhas educativas e orientações pós-trauma direcionadas aos pacientes, além de capacitações para os cirurgiões-dentistas devem ser realizadas a fim de obter menor frequência de traumatismos dentários e consequentemente de sequelas.

\section{REFERÊNCIAS}

1. Cortes MI, Marcenes W, Sheiham A. Impact of traumatic injuries to the permanent teeth on the oral health-related quality of life in 12-14-year-old children. Community Dent Oral Epidemiol. 2002; 30(3):193-8.

2. Azami-Aghdash S, Ebadifard Azar F, Pournaghi Azar F, Rezapour A, Moradi-Joo M, Moosavi A, et al. Prevalence, etiology, and types of dental trauma in children and adolescents: systematic review and metaanalysis. Med J Islam Repub Iran. 2015; 29(4):234.
3. Granville-Garcia AF, de Menezes VA, de Lira PI Dental trauma and associated factors in Brazilian preschoolers. Dent Traumatol. 2006; 22(6):318-22.

4. Toprak ME, Tuna EB, Seymen F, Gençay K. Traumatic dental injuries in Turkish children, Istanbul. Dent Traumatol. 2014; 30(4):280-4.

5. Díaz JA, Bustos L, Brandt AC, Fernández BE. Dental injuries among children and adolescents aged 1-15 years attending to public hospital in Temuco, Chile. Dent Traumatol. 2010; 26(3):254-61.

6. Kovacs M, Pacurar M, Petcu B, Bukhari C. Prevalence of traumatic dental injuries in children who attended two dental clinics in Targu Mures between 2003 and 2011. Oral Health Dent Manag. 2012; 11(3):116-24.

7. Olsburgh S, Jacoby T, Krejci I. Crown fractures in the permanent dentition: pulpal and restorative considerations. Dent Traumatol. 2002; 18(3):103-15

8. Love RM, Jenkinson HF. Invasion of dentinal tubules by oral bacteria. Crit Rev Oral Biol Med. 2002; 13(2):17183.

9. Breik O. Discuss how the management of trauma to the dentition is influenced by the type and severity of injury. Aust Endod J. 2008; 34(3):120-5.

10. Ravn JJ. Follow-up study of permanent incisors with enamel-dentin fractures after acute trauma. Scand J Dent Res. 1981; 89(5):355-65.

11. Andreasen FM, Pedersen BV. Prognosis of luxated permanent teeth - the development of pulp necrosis. Endod Dent Traumatol. 1985; 1(6):207-20.

12. Bakland LK, Andreasen JO. Dental traumatology: essencial diagnosis and treatment planning. Endod Topics. 2004; 7(1):14-34.

13. Andreasen JO. Luxation of permanent teeth due to trauma. A clinical and radiographic follow-up study of 189 injured teeth. Scand J Dent Res. 1970; 78(3):27386.

14. Andreasen FM. Pulpal healing after luxation injuries and root fracture in the permanent dentition. Endod Dent Traumatol. 1989; 5(3):111-31.

15. Andreasen JO, Andreasen FM. Texto e atlas colorido de traumatismo dental. 3. ed. Porto Alegre: Artmed; 2001.

16. Jacobsen I. Criteria for diagnosis of pulp necrosis in traumatized permanent incisors. Scand J Dent Res. 1980; 88(4):306-12.

17. Andreasen FM, Zhijie Y, Thomsen BL. Relationship between pulp dimensions and development of pulp necrosis after luxation injuries in the permanent dentition. Endod Dent Traumatol. 1986; 2(3):90-8.

18. Stockwell AJ. Incidence of dental trauma in the Western Australian School Dental Service. Community Dent Oral Epidemiol. 1988; 16(5):294-8.

19. Gábris K, Tarján I, Rózsa N. Dental trauma in children presenting for treatment at the Department of Dentistry for Children and Orthodontics, Budapest, 1985-1999. Dent Traumatol. 2001; 17(3):103-8.

20. Taiwo OO, Jalo HP. Dental injuries in 12-year old Nigerian students. Dent Traumatol. 2011; 27(3):230-4.

21. Cavalleri G, Zerman N. Traumatic crown fractures in permanent incisors with immature roots: a follow-up study. Endod Dent Traumatol. 1995; 11(6):294-6.

22. Hecova H, Tzigkounakis V, Merglova V, Netolicky J. A retrospective study of 889 injured permanent teeth. Dent Traumatol. 2010; 26(6):466-75.

23. Wang C, Qin M, Guan Y. Analysis of pulp prognosis in 603 permanent teeth with uncomplicated crown fracture 
with or without luxation. Dent Traumatol. 2014; 30(5): 333-7.

24. Robertson A, Andreasen FM, Andreasen JO, Norén JG. Long-term prognosis of crown-fractured permanent incisors. The effect of stage of root development and associated luxation injury. Int $\mathbf{J}$ Paediatr Dent. 2000;10(3):191-9.

25. Andreasen FM, Kahler B. Pulpal response after acute dental injury in the permanent dentition: clinical implications-a review. J Endod. 2015; 41(3):299-308.

\section{CONFLITO DE INTERESSES}

Os autores declaram não haver conflitos de interesse.

\section{AUTOR PARA CORRESPONDÊNCIA}

Marcos Sérgio Endo

marcossendo@gmail.com

Submetido em 13/03/2017

Aceito em 02/05/2017 